\title{
Low Velocity Impact Localization System Using FBG Array and MVDR Beamforming Algorithm
}

\author{
Yaozhang SAI, Mingshun JIANG ${ }^{*}$, Qingmei SUI, Lei JIA, and Shizeng LU \\ School of Control Science and Engineering, Shandong University, Jinan, 250100, China \\ *Corresponding author: Mingshun JIANGＥ-mail: jiangmingshun@sdu.edu.com
}

\begin{abstract}
This paper proposes an impact localization system based on the fiber Bragg grating (FBG) array and minimum variance distortionless response (MVDR) beamforming algorithm. The linear FBG array, which contains seven FBG sensors, is used for detecting impact signals. Morlet wavelet transform is applied for extracting narrow-band signals of impact signals. According to the MVDR beamforming algorithm, the system realizes single-impact and multi-impact localizations. The localization system is verified on a $500 \mathrm{~mm} \times 500 \mathrm{~mm} \times 2 \mathrm{~mm}$ carbon fiber reinforced polymer (CFRP) plate for single-impact and multi-impact localizations. The average locating error and the maximum locating error are $6.8 \mathrm{~mm}$ and $9.9 \mathrm{~mm}$, respectively.
\end{abstract}

Keywords: Fiber Bragg grating, minimum variance distortionless response, Morlet wavelet transform, multi-impact localization

Citation: Yaozhang SAI, Mingshun JIANG, Qingmei SUI, Lei JIA, and Shizeng LU, "Low Velocity Impact Localization System Using FBG Array and MVDR Beamforming Algorithm," Photonic Sensors, 2015, 5(4): 357-364.

\section{Introduction}

For high-performance and lightweight structures, the increasing demands from aircraft industry stimulate application and development of composite materials $[1,2]$. In the practical application of composite structure, impact can cause various barely visible damages, such as matrix cracking, ply delamination, and fiber fracture $[3,4]$. The damages seriously degrade the security of composite structure $[5,6]$. Therefore, impact event should be timely localized for the damage detection. Many methods have been researched for impact localization. But the localization object is mainly single-impact. In practice, multiple impact events often appear at the same time. Hence, the multi-impact localization method is imperative for the composite structure.
Due to light weight and immunity to electromagnetic interference, fiber optic sensors have been widely researched for impact localization on the composite structure. Kirkby et al. [7] applied a triangle technology to locate impact source on composite panels by using fiber Bragg grating (FBG) sensors. Fu et al. [8] used the hyperbolic curves algorithm and four fiber optic sensors to achieve impact localization on the composite plate. Frieden et al. [9] obtained the location of impact through interpolation of a reference data set, consisting of arrival time delays and known location. The methods need the arrival time of impact signals. When multiple impact events simultaneously appear, the mixed impact signals of different impact sources need to be distinguished for obtaining arrival time of impact signals and locating multi-impact. Riberiro et

Received: 27 July 2015 / Revised: 25 August 2015

(C) The Author(s) 2015. This article is published with open access at Springerlink.com

DOI: $10.1007 / \mathrm{s} 13320-015-0271-y$

Article type: Regular 
al. [10] and Jang et al. [11] used FBG sensors and the neural network method to obtain the location of impact source on the composite structure, respectively. Lu et al. [12] proposed support vector regression to determine impact source on the composite structure by using FBGs. Jiang et al. [13] used the extreme learning machine and FBG sensor network to obtain the location of impact. Lu et al. [14] applied least squares support vector machines and FBG sensors to localize impact. These methods can realize multi-impact localization, but a large number of training samples are needed before impact localization.

This paper proposes a multi-impact localization system based on the FBG array and minimum variance distortionless response (MVDR) beamforming algorithm. A high speed FBG interrogation system is designed to detect impact signals. Morlet wavelet transform is applied to extract narrow-band signals of impact signals. MVDR beamforming algorithm is applied to localize multi-impact by the impact signals of FBG array. The system and localization algorithm are verified on a composite plate. The paper provides a novel multi-impact localization algorithm.

\section{Localization algorithm}

\subsection{MVDR beamforming algorithm}

$N$ sensors compose a line sensor array. The spacing of the neighboring sensors is $d$, as shown in Fig. 1. The coordinate of Sensor $S_{i}$ is $(i d, 0)$ $(i=0,1, \cdots, N)$. Assuming that $K$ impacts occur, the coordinate of impact is $\left(x_{k}, y_{k}\right)(k=1,2, \cdots, K)$. The output signal of Sensor $S_{i}$ can be expressed as

$$
z_{i}(t)=\sum_{k=1}^{K} s_{k i}(t)+n_{i}(t)
$$

where $s_{k i}(t)$ and $n_{i}(t)$ are the impact signal of the $k$ th impact and noise signal, respectively. $S_{0}$ is considered as the reference sensor. The $k$ th impact causes the elastic waves with a certain frequency component $\omega$. The impact signal of the $k$ th impact source, which is received by Sensor $S_{i}$, can be represented by

$$
s_{k i}(t)=\frac{r_{k 0}}{r_{k i}} S_{k 0}(t) e^{-\mathrm{j} \omega \tau_{k i}}
$$

where $\tau_{k i}$ is the arrival time difference between $S_{0}$ and $S_{i}, r_{k i}$ is the distance from the $k$ th impact source to $S_{i}$, and $S_{k 0}(t)$ is the impact signal which is received by $S_{0}$ and comes from the $k$ th impact source. It can be expressed as

$$
\tau_{k i}=\frac{r_{k i}-r_{k 0}}{c}=\frac{\sqrt{\left(x_{k}-(i d)\right)^{2}+y_{k}^{2}}-\sqrt{x_{k}^{2}+y_{k}^{2}}}{c}
$$

where $c$ is the wave velocity. Let $\mathbf{a}\left(x_{k}, y_{k}\right)$ denote the steering vector. It is represented as

$$
a_{k i}\left(x_{k}, y_{k}\right)=\frac{r_{k 0}}{r_{k i}} e^{-j \omega \tau_{k i}} \text {. }
$$

For the whole sensor array, the signal vector can be expressed as

$$
\mathbf{Z}(t)=\mathbf{A}(x, y) \mathbf{s}(t)+\mathbf{N}(t)
$$

where

$$
\begin{gathered}
\mathbf{Z}(t)=\left[z_{1}(t), z_{2}(t), \cdots, z_{N}(t)\right]^{T} \\
\mathbf{A}(x, y)=\left[\mathbf{a}\left(x_{1}, y_{1}\right), \mathbf{a}\left(x_{2}, y_{2}\right), \cdots, \mathbf{a}\left(x_{K}, y_{K}\right)\right] \\
\mathbf{s}(t)=\left[s_{1}(t), s_{2}(t), \cdots, s_{K}(t)\right]^{T} \\
\mathbf{N}(t)=\left[n_{1}(t), n_{2}(t), \cdots, n_{N}(t)\right]^{T} .
\end{gathered}
$$

The steering vector $\mathbf{a}\left(x_{k}, y_{k}\right)$ can be given by $\mathbf{a}\left(x_{k}, y_{k}\right)=\left[a_{k 1}\left(x_{k}, y_{k}\right), a_{k 2}\left(x_{k}, y_{k}\right), \cdots, a_{k N}\left(x_{k}, y_{k}\right)\right]^{T}$.

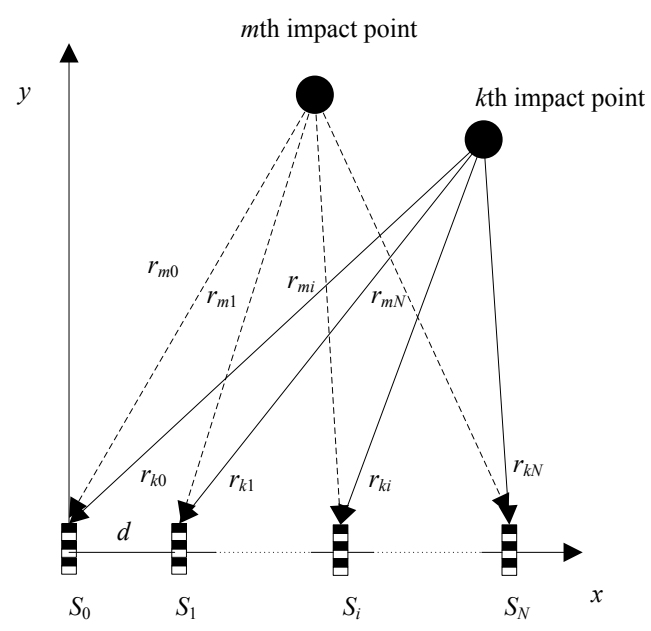

Fig. 1 Localization algorithm. 
The essence of beamforming is to linearly combine the signals from sensors in a manner, that is, with a certain weighting, examining signals arriving from a specific location $[15,16]$. The beamforming process is shown in Fig. 2.

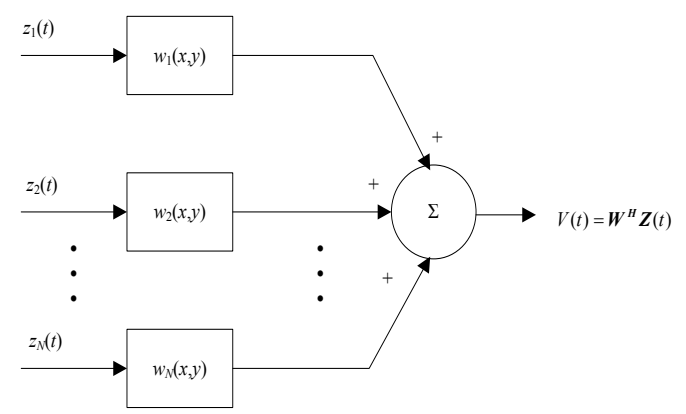

Fig. 2 Beamforming algorithm.

As for the linear array with $N$ sensors introduced above, the output of beamforming can be expressed as

$$
V(t)=\mathbf{W}^{H} \mathbf{Z}(t)
$$

where $\mathbf{W}=\left[w_{1}, w_{2}, \cdots, w_{N}\right]^{T}$ is the weighted vector. The output power of the linear array is

$$
P=E\left[V(t) V^{H}(t)\right]=\mathbf{W}^{H} \mathbf{R W}
$$

where $\mathbf{R}=E\left[\mathbf{Z}(t) \mathbf{Z}^{H}(t)\right]$ is the covariance matrix of signals of the sensor array.

The objective of the MVDR beamforming algorithm is to preserve the power output of the impact signal and minimize the power output of interference and noise $[17,18]$. Then the essence is a question of minimizing the constraint:

$$
\min \mathbf{W}^{H} \mathbf{R W} \text { to subject } \mathbf{W}^{H} \mathbf{a}(x, y)=1
$$

where $\mathbf{a}(x, y)$ is the steering vector. The solution of the above problem is given by

$$
\mathbf{W}_{\mathrm{MVDR}}=\frac{\mathbf{R}^{-1} \mathbf{a}(x, y)}{\mathbf{a}^{H}(x, y) \mathbf{R}^{-1} \mathbf{a}(x, y)} .
$$

According to (8) and (10), the output power of MVDR is

$$
P_{\mathrm{MVDR}}(x, y)=\frac{1}{\mathbf{a}^{H}(x, y) \mathbf{R}^{-1} \mathbf{a}(x, y)} .
$$

In practical applications, $\mathbf{R}$ is estimated by a finite number of data vectors:

$$
\hat{\mathbf{R}}=\frac{1}{L} \mathbf{Z Z}^{H}
$$

where $\hat{\mathbf{R}}$ is the estimation of $\mathbf{R}$, and $L$ is the number of the snapshots. Hence, the output power can be expressed as

$$
P_{\mathrm{MVDR}}(x, y)=\frac{1}{\mathbf{a}^{H}(x, y) \hat{\mathbf{R}}^{-1} \mathbf{a}(x, y)} .
$$

When the MVDR beamforming algorithm is used for impact localization, the monitoring area is divided into a large number of points. The steering vector $\mathbf{a}(x, y)$ of each point is obtained. According to the covariance matrix $\hat{\mathbf{R}}$ of impact signals of sensor array and (13), the output power of each point is calculated. Based on the values of output powers, localization imaging is compounded. The location of peak of output power is the impact point.

\subsection{Morlet wavelet transform}

The MVDR beamforming algorithm requires that the impact signal is a narrow-band signal. The elastic waves caused by impact are wide-band signals. Therefore, the Morlet wavelet transform is introduced for extracting the narrow-band signal of the impact signal. Moreover, the arrival time difference of impact signals of different sensors is obtained by the Morlet wavelet transform. According to the arrival time difference, the wave velocities in different directions are calculated on the composite material.

The Morlet wavelet transform of impact signals $u(x, t)$ is expressed as

$$
\mathbf{W} T(x, a, b)=\frac{1}{\sqrt{a}} \int_{-\infty}^{+\infty} u(x, t) \psi^{*}\left(\frac{t-b}{a}\right) d t
$$

where $\psi^{*}(t)$ is the complex conjugate of the mother wavelet, $a$ is the scale factor, and $b$ is the time factor. The Morlet wavelet is given by [19]

$$
\psi(t)=\frac{1}{\sqrt{\pi f_{b}}} e^{2 j \pi f_{c} t} e^{-\frac{t^{2}}{f_{b}}} .
$$

The Fourier transform is given by

$$
\Psi(\omega)=e^{-\frac{\omega_{b}}{8 \pi}\left(\omega-\omega_{c}\right)^{2}}
$$

where $f_{b}=\omega_{b} / 2 \pi$ is the wavelet bandwidth, and $f_{c}=\omega_{c} / 2 \pi$ is the center frequency. The function can be considered as a modulated Gaussian function. 
Therefore, the narrow band signal, whose central frequency is $\omega_{c}$, is extracted by the Morlet wavelet transform. The bandwidth is limited in the range of $\left[\left(\omega_{c}-\omega_{b}\right) / 2,\left(\omega_{c}+\omega_{b}\right) / 2\right]$. The impact signal $u(x, t)$ can be considered as a time harmonic motion of two signals of unit amplitude with different frequencies $\omega_{1}$ and $\omega_{2}$ propagating in the $x$-direction as follows:

$$
u(x, t)=e^{-\mathrm{j}\left(k_{1} x-\omega_{1} t\right)}+e^{-\mathrm{j}\left(k_{2} x-\omega_{2} t\right)}
$$

where $k_{1}$ and $k_{2}$ are the wave numbers. Introduction:

$$
\begin{aligned}
& \Psi^{*}(a \omega)=\Psi^{*}\left(a \omega_{1}\right)=\Psi^{*}\left(a \omega_{2}\right) \\
& \frac{k_{2}-k_{1}}{2}=\Delta k, \frac{\omega_{2}-\omega_{1}}{2}=\Delta \omega \\
& \frac{k_{1}+k_{2}}{2}=k_{0}, \frac{\omega_{1}+\omega_{2}}{2}=\omega_{0} .
\end{aligned}
$$

The module value of impact signal is acquired by the Morlet wavelet transform:

$$
|\mathbf{W} T(x, a, b)|=\sqrt{2 a}\left|\Psi\left(a \omega_{0}\right)\right| \sqrt{1+\cos (\Delta \omega b-\Delta k x)} .
$$

When $b=\Delta k / \Delta \omega$, the module value is the maximum. The time difference of impact signals of different sensors are obtained by the peak time of module values.

\section{Localization experiments}

\subsection{Experimental setup}

The experimental setup contains a carbon fiber reinforced polymer (CFRP) plate with a dimension of $500 \mathrm{~mm} \times 500 \mathrm{~mm} \times 2 \mathrm{~mm}$. The ply sequence is $[45 / 0 /-45 / 90 / 0 /-45 / 0 /-45 / 0] \mathrm{s}$. Four edges of the plate are clamped tightly by metal fixture. The FBG sensor is used for detecting the impact signal. The length and reflectivity of the FBG are $3 \mathrm{~mm}$ and $70 \%$, respectively. The wavelengths of FBGs are within the limits of $1550 \pm 0.1 \mathrm{~nm}$. The high speed FBG interrogation system comprises amplified spontaneous emission (ASE) source, edge-filter, couplers, photoelectric detectors (PD), amplifier (AMP), and data acquisition equipment with sampling frequency of $5 \mathrm{mHz}$. The wavelengths parameters of FBGs all lie in the edge range of wavelength of ASE source. Power demodulation method, based on edge filter principle [20, 21], satisfies the need of acquiring high frequency signals. Impact events are generated by steel balls with the diameter of $20 \mathrm{~mm}$.

\subsection{Wave velocities measurement}

According to (3), the MVDR beamforming algorithm needs wave velocity for impact localization. Due to the anisotropy of composite material, the wave velocities of different directions are different. Therefore, the wave velocities of different directions should be measured for the MVDR beamforming algorithm.

Four FBGs are stuck on the CFRP plate, as shown in Fig. 3. The coordinates of FBGs are (150, $0),(0,-150),(-150,0)$, and $(0,150)$, respectively. The high speed FBG interrogation system is shown in Fig. 4. Impact experiment is preformed on A point first. The angle between $\mathrm{AS}_{1}$ and $x$ axis is $30^{\circ}$. Impact signals of $S_{2}$ and $S_{4}$ are shown in Fig. 5. Figure 6 is the frequency spectrum of the signal of $S_{2}$. The impact signal is a wide-band signal. The frequency band mainly is from $0 \mathrm{kHz}$ to $100 \mathrm{kHz}$. The narrow-band signals, whose central frequency is $45 \mathrm{kHz}$, are extracted by the Morlet wavelet transform. The arrival time difference of the signals of $S_{2}$ and $S_{4}$ is obtained by the peaks of module values of the narrow-band signals, as shown in Fig. 7. According to the distance difference between $\mathrm{AS}_{2}$ and $\mathrm{AS}_{4}$ and the arrival time difference of the signals of $S_{2}$ and $S_{4}$, the wave velocity $\left(c_{90}\right)$ of $90^{\circ}$ direction is calculated. The arrival time difference $(\Delta t)$ of the signals of $S_{1}$ and $S_{2}$ is extracted. The wave velocity $\left(c_{30}\right)$ of $30^{\circ}$ direction is obtained by

$$
\frac{L_{S_{1} A}}{c_{30}}-\frac{L_{S_{2} A}}{c_{90}}=\Delta t
$$

According to the above method, the wave velocities of the other directions are calculated, as shown in Fig. 8. 


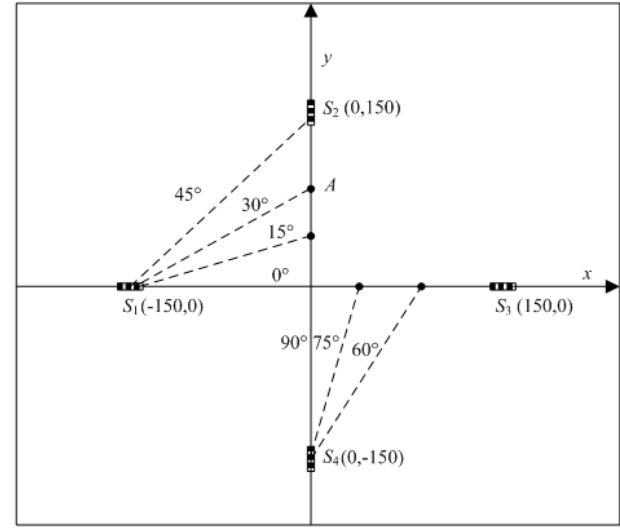

Fig. 3 Wave velocities measurement.

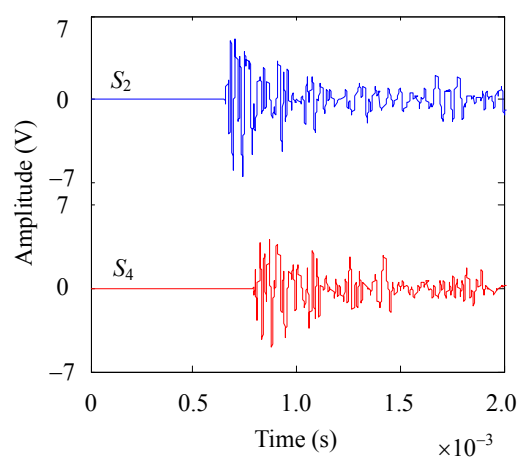

Fig. 5 Impact signals of $S_{2}$ and $S_{4}$.

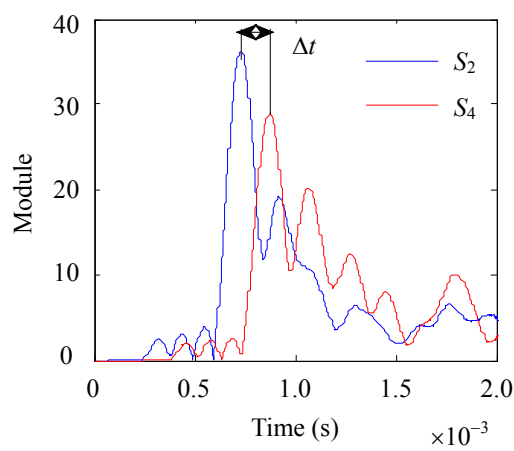

Fig. 7 Time difference of narrow-band signals.

\subsection{Localization experiments}

Localization experiments are performed in $400 \mathrm{~mm} \times 400 \mathrm{~mm}$ monitoring area. Seven FBGs are stuck on $x$ axis, as shown in Fig.9. The coordinate of FBG $S_{4}$ is $(0,0)$. The spacing of the neighboring FBGs is $10 \mathrm{~mm}$. The signal demodulation system is consistent with Fig. 4. The system includes the ASE source, edge-filter, couplers, PD, AMP, and data acquisition equipment. A single-impact experiment is performed on $(-85,229)$ which is randomly

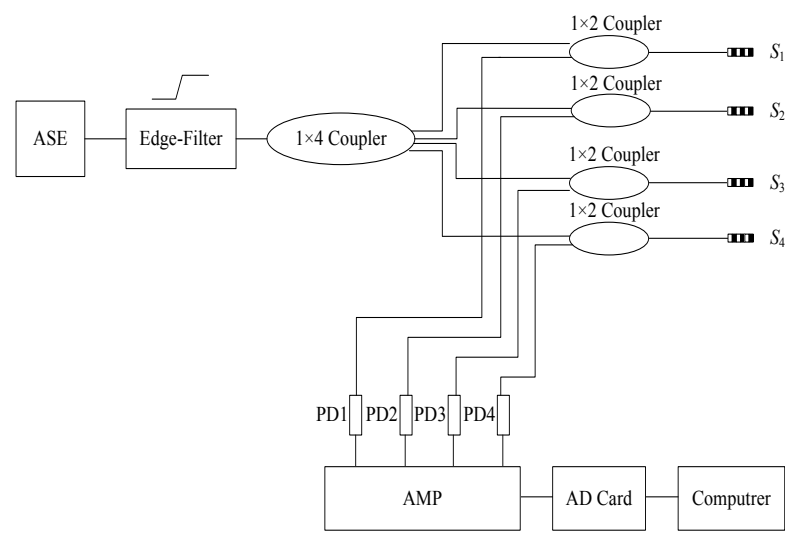

Fig. 4 Interrogation system.

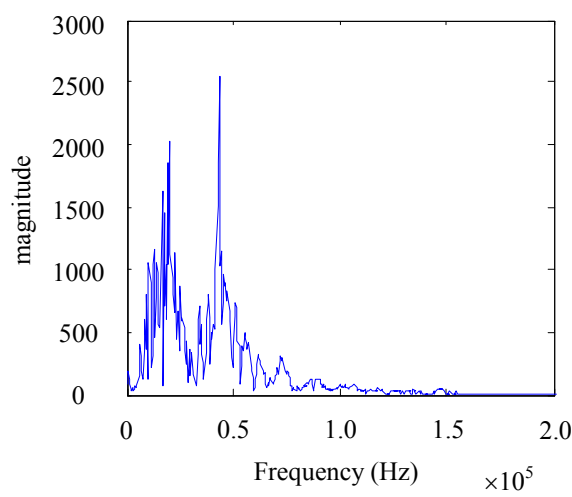

Fig. 6 Frequency spectrum of impact signal.

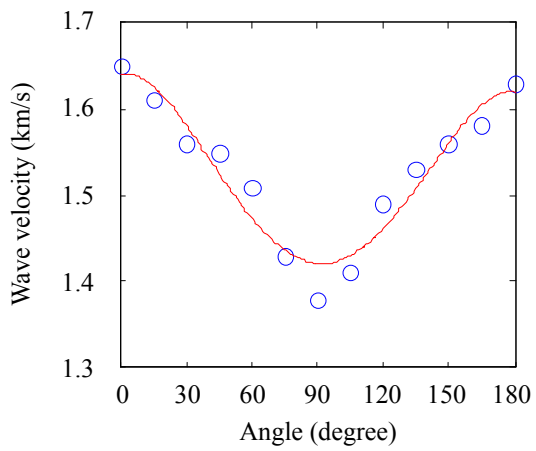

Fig. 8 Wave velocities of different directions.

selected. The impact signals of FBG array are shown in Fig. 10. According to the Morlet wavelet transform, the narrow-band signals of impact signals are extracted, as shown in Fig. 11. The monitoring area is divided into 160000 points. The steering vector $\mathbf{a}(x, y)$ of each point is calculated. According to (13), the output power of MVDR of each point is calculated. The output powers are considered as pixel values. Then the localization image is obtained, as shown in Fig. 12. The 
coordinate of the maximum output power is impact point. The coordinate of the single-impact experiment is $(-89,232)$. To evaluate the accuracy of the results, an error function is defined as

$$
\varepsilon=\sqrt{\left(x_{\text {actual }}-x_{\text {predicted }}\right)^{2}+\left(y_{\text {actual }}-y_{\text {predicted }}\right)^{2}}
$$

where $\left(x_{\text {actual }}, y_{\text {actual }}\right)$ is the coordinate of actual impact source and $\left(x_{\text {predicted }}, y_{\text {predicted }}\right)$ is the coordinate of predicted impact source. Hence, the localization error of the single-impact is $5.7 \mathrm{~mm}$.

Dual-impact events are randomly selected to verify the multi-impact localization. The coordinates of dual-impact are $(53,65)$ and $(167,361)$. The impact signals and narrow-band signals are shown in Figs. 13 and 14, respectively. According to the MVDR beamforming algorithm, the multi-impact localization is shown in Fig. 15. The predicted coordinates of dual-impact are $(59,67)$ and $(171$, 367). The localization errors are $6.3 \mathrm{~mm}$ and $7.2 \mathrm{~mm}$, respectively.

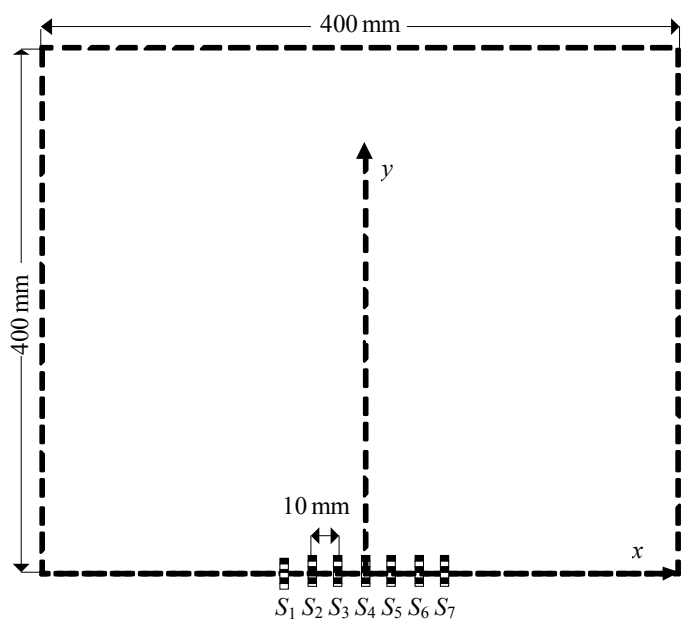

Fig. 9 Localization experiment.

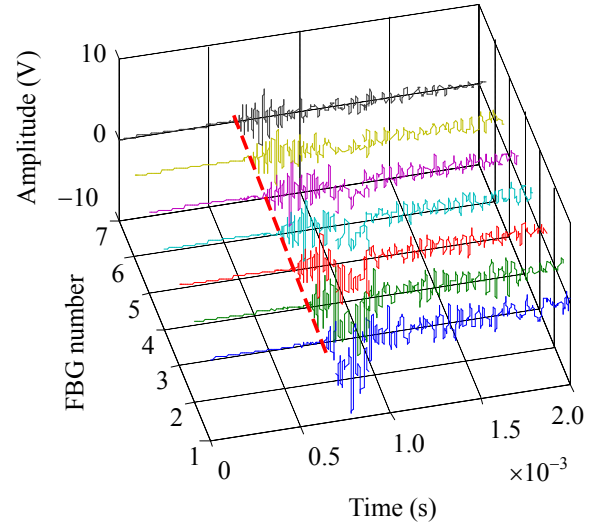

Fig. 10 Impact signals of single-impact.

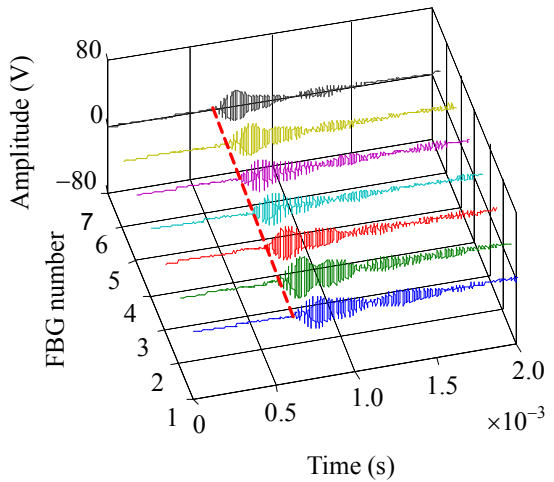

Fig. 11 Narrow-band signals of single-impact.

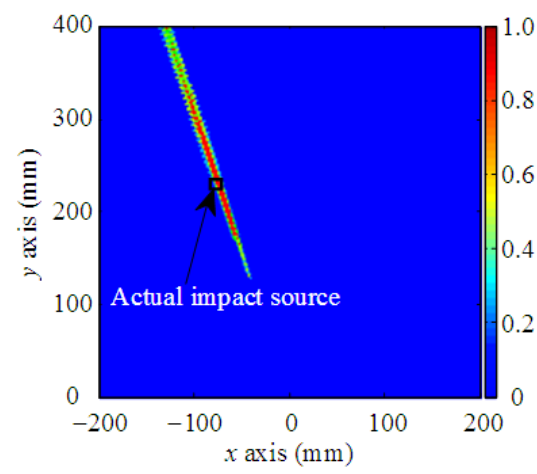

Fig. 12 Single-impact localization.

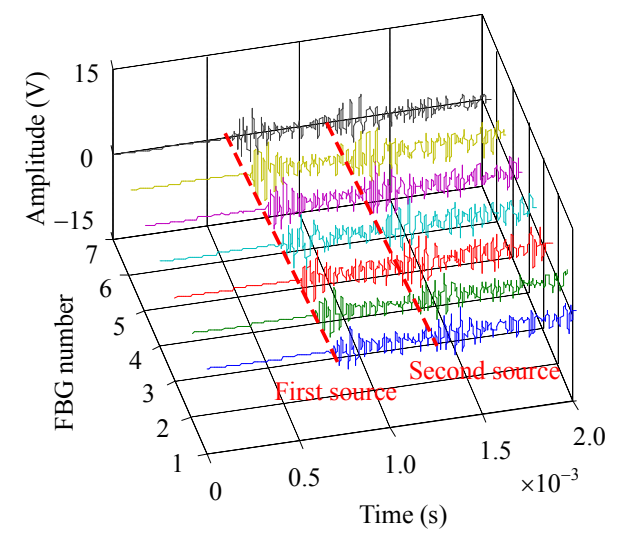

Fig. 13 Impact signals of dual-impact.

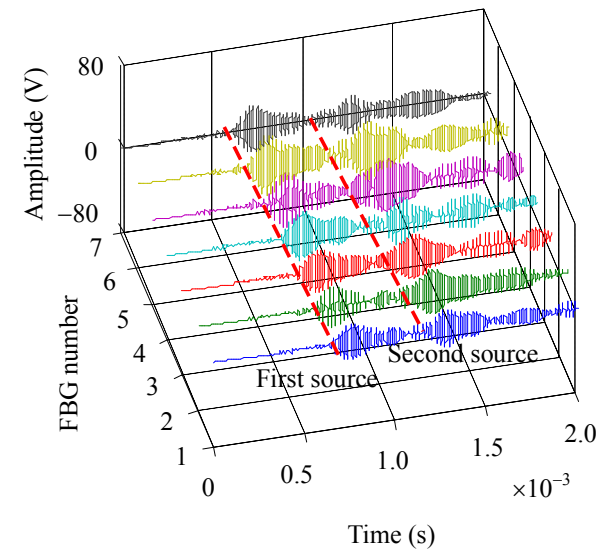

Fig. 14 Narrow-band signals of dual-impact. 


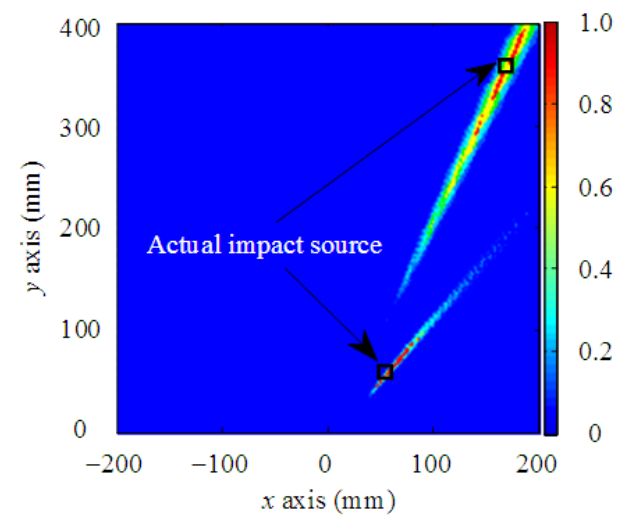

Fig. 15 Dual-impact localization.

In order to further verify the capacity of the multi-impact localization of the MVDR beamforming algorithm, five dual-impact experiments are performed. The results of localization experiments are shown in Table 1. The average error and the maximum error are $6.8 \mathrm{~mm}$ and $9.9 \mathrm{~mm}$, respectively. The experiments demonstrate that MVDR beamforming algorithm can determine location of impacts.

Table 1 Localization results.

\begin{tabular}{ccccccc}
\hline $\begin{array}{c}\text { Impact } \\
\text { event }\end{array}$ & \multicolumn{2}{c}{$\begin{array}{c}\text { Actual coordinate } \\
(\mathrm{mm})\end{array}$} & $\begin{array}{c}\text { Predicted coordinate } \\
(\mathrm{mm})\end{array}$ & $\begin{array}{c}\text { Error } \\
(\mathrm{mm})\end{array}$ \\
\hline 1 & $(-181,76)$ & $(-136,238)$ & $(-178,80)$ & $(-133,236)$ & 5 & 3.6 \\
2 & $(-65,273)$ & $(-58,110)$ & $(-69,279)$ & $(-63,116)$ & 7.2 & 7.8 \\
3 & $(52,79)$ & $(131,196)$ & $(46,75)$ & $(137,203)$ & 8.1 & 9.2 \\
4 & $(178,89)$ & $(151,259)$ & $(179,93)$ & $(158,262)$ & 4.1 & 7.6 \\
5 & $(128,113)$ & $(-139,197)$ & $(121,106)$ & $(-136,192)$ & 9.9 & 5.8 \\
\hline
\end{tabular}

\section{Conclusions}

The paper proposes an impact localization system based on the FBG network and MVDR beamforming algorithm. An FBG linear array is utilized for detecting impact signals. The edge-filter method is used for signal interrogation. The Morlet wavelet transform is mainly applied for extracting the narrow-band signals of impact signals. The MVDR beamforming algorithm is employed for impact localization. The localization system is verified on $500 \mathrm{~mm} \times 500 \mathrm{~mm} \times 2 \mathrm{~mm}$ CFRP plate for single-impact and multi-impact localizations. The average localization error and the maximum localization error are $6.8 \mathrm{~mm}$ and $9.9 \mathrm{~mm}$, respectively. The experiments demonstrate that the localization system can localize the multi-impact. Hence, it can meet the requirements of practical multi-impact localization.

\section{Acknowledgment}

This research is partially supported by the National Natural Science Foundation of China (Grant no. 61174018) and Fundamental Research Funds of Shandong University (Grant no. 2014YQ009).

Open Access This article is distributed under the terms of the Creative Commons Attribution License which permits any use, distribution, and reproduction in any medium, provided the original author(s) and source are credited.

\section{References}

[1] M. Grujicic, B. Pandurangan, W. C. Bell, C. F. Yen, and B. A. Cheeseman, "Application of a dynamic-mixture shock-wave model to the metal-matrix composite materials," Materials Science and Engineering: A, 2011, 528(28): 8187-8197.

[2] A. Katunin, K. Dragan, and M. Dziendzikowski, "Damage identification in aircraft composite structures: A case study using various non-destructive testing techniques," Composite Structures, 2015, 127(9): 1-9.

[3] F. Otero, S. Oller, S. X. Martinez, and O. Salomon, "Numerical homogenization for composite materials analysis. Comparison with other micro mechanical formulations," Composite Structures, 2015, 122(4): 405-416.

[4] H. Singh, K. K. Namala, and P. Mahajan, "A damage evolution study of E-glass/epoxy composite under low velocity impact," Composites Part B: Engineering, 2015, 76(8): 235-248.

[5] J. Zhang and X. Zhang, "An efficient approach for predicting low-velocity impact force and damage in composite laminates," Composite Structures, 2015, 130(12): 85-94.

[6] P. Yang, S. S. Shams, A. Slay, B. Brokate, and R. Elhajjar, "Evaluation of temperature effects on low velocity impact damage in composite sandwich panels with polymeric foam cores," Composite Structures, 2015, 129(11): 213-223.

[7] E. Kirkby, R. d. Oliveira, V. Michaud, and J. A. Manson, "Impact localisation with FBG for a self-healing carbofibre composite structure," Composite Structures, 2011, 94(1): 8-14. 
[8] T. Fu, Y. Liu, K. Lau, and J. Leng, "Impact source identification in a carbon fiber reinforced polymer plate by using embedded fiber optic acoustic emission sensors," Composites Part B: Engineering, 2014, 66(11): 420-429.

[9] J. Frieden, J. Cugnoni, J. Botsis, and T. Gmur, "Low energy impact damage monitoring of composites using dynamic strain signals from FBG sensors - Part I: Impact detection and localization," Composite Structures, 2012, 94(2): 438-445.

[10]F. Ribeiro, G. R. Collere Possetti, J. L. Fabris, and M. Muller, "Smart optical fiber sensor for impact localization on planar structures," in 2013 SBMO/IEEE MTT-S International Microwave \& Optoelectronics Conference (IMOC), Rio de Janeiro, pp. 1-3, 2013.

[11] B. W. Jang, Y. G. Lee, J. H. Kim, Y. Y. Kim, and C. G. Kim, "Real-time impact identification algorithm for composite structures using fiber Bragg grating sensors," Structural Control and Health Monitoring, 2012, 19(7): 580-591.

[12] J. Lu, B. Wang, and D. Liang, "Wavelet packet energy characterization of low velocity impacts and load localization by optical fiber Bragg grating sensor technique," Applied Optics, 2013, 52(11): 2346-2352.

[13] M. Jiang, S. Lu, Q. Sui, H. Dong, Y. Sai, and L. Jia, "Low velocity impact localization on CFRP based on FBG sensors and ELM algorithm," IEEE Sensors Journal, 2015, 15(8): 4451-4456.

[14] S. Lu, M. Jiang, Q. Sui, Y. Sai, and L. Jia, "Low velocity impact localization system of CFRP using fiber Bragg grating sensors," Optical Fiber Technology, 2015, 21(1): 13-19.

[15] J. Chang, "A robust adaptive array beamformer using particle swarm optimization for space-time code division multiple access systems," Information Sciences, 2014, 278(25): 174-186.

[16] T. N. Tran, W. Cowley, and A. Pollok, "Automatic adaptive speech separation using beamformer-output-ratio for voice activity classification," Signal Processing, 2015, 113(8): 259-272.

[17] Y. Zeng and R. C. Hendriks, "Distributed estimation of the inverse of the correlation matrix for privacy preserving beamforming," Signal Processing, 2015, 107(2), 109-122.

[18] D. Feng, X. Li, H. Lv, H. Liu, and Z. Bao, "Two-sided minimum-variance distortionless response beamformer for MIMO radar," Signal Processing, 2009, 89(3): 328-332.

[19] K. C. Gryllias and I. A. Antoniadis, "Estimation of the instantaneous rotation speed using complex shifted Morlet wavelets," Mechanical Systems and Signal Processing, 2013, 38(1): 78-95.

[20] D. Pang and Q. Sui, "Response analysis of ultrasonic sensing system based on fiber bragg gratings of different lengths," Photonic Sensors, 2014, 4(3): 281-288.

[21] Z. Jin, M. Shun, Q. Sui, F. Zhang, and L. Jia, "Acoustic emission source linear localization based on an ultra-short FBGs sensing system," Photonic Sensors, 2014, 4(2): 152-155. 